\title{
Acute subdural empyema in the young COVID-19 patient- $A$ case report
}

\author{
Haroon $\mathrm{K}^{1}$, Reza $\mathrm{MA}^{2}$, Taher $\mathrm{T}^{3}$, Alam $\mathrm{MS}^{4}$, Haque $\mathrm{RU}^{5}$, Ahmed $\mathrm{MF}^{6}$, Hossain $\mathrm{SS}^{7}$ \\ Conflict of interest: There is no conflict \\ of interest relevant to this paper to \\ disclose. \\ Funding Agency : was not funded by \\ any institute or any group \\ Contribution of Authors : Principal \\ Investigator- Dr. Kaisar Haroon

\begin{abstract}
:
Acute subdural empyema is a surgical emergency. It is life threatening for the patient. It has to be evacuated as soon it is diagnosed. But subdural empyema in a COVID 19 patient is uncommon. Its management put the surgical team to a new dilemma. On one side the patient's life was at risk and on the other side the whole surgical team might get infected.In this case report, we describe such a case which saved the patient's life at same time many doctors, nurses and OT attendants became infected.
\end{abstract}

Manuscript preparation- Md.Shafiul

Alam, Md. Arif Reza, Tania Taher

Data collection- Rakib-Ul-Haque, Md.

Farhad Ahmed

Editorial formatting-Sk. Sader Hossain.

Copyright: @2020bang.BJNS published by BSNS. This article is published under the creative commons CC-BY-NC

license. This license permits use distribution (https://creativecommons. orgf/licences/by-nc/4-0/)reproduction in any medium, provided the original work is properly cited and is not used for commercial purposes.

Received: $15 / 10 / 2020$

Accepted: 30/10/2020
Abbrevaitions: OPD- Out patient department, COVID 19-Corona Virus Disease 2019, NINS- National Institute of Neurosciences, RT-PCR-Reverse transcription polymerase chain reaction. SDE- Subdural empyema, PNS- para nasal sinuses

Key Words: COVID 19, Coronavirus, SARSCOV2, subdural empyema

Bang. J Neurosurgery 2021; 10(2): 206-209

\section{Introduction:}

Subdural empyema (SDE) is a rare but potentially lifethreatening disease consisting of a pyogenic infection in the pre-formed space between the inner surface of the dura mater and the outer surface of the arachnoid layer. ${ }^{1}$ The potential subdural space is relatively free over and between the cerebral hemispheres but restricted in the central basal region; hence most subdural empyemas are found either on the convexity or in the parafalx region. ${ }^{2}$ Contrary to the typical convexity SDEs, the tendency of pus to extend along the length of the falx below the longitudinal sinus and bridging veins makes parafalcine, or interhemispheric, SDEs relatively difficult-to-reach collections. ${ }^{1}$

Headache, hemiparesis, pyrexia, meningism, epilepsy, and vomiting were the commonest presenting features, and at least four of these were found in the majority of patients. ${ }^{3}$

1. Dr. Kaisar Haroon, Assistant Professor, Department of Neurosurgery, NINSH

2. Dr. Md. Arif Reza, Assistant Professor, Department of Neurosurgery, NINSH

3. Dr. Tania Taher, Assistant Professor, HFRC Medical College Hospital

4. Dr. Md.Shafiul Alam, Associate Professor, Department of Neurosurgery, NINSH

5. Dr. Rakib-Ul-Haque, Associate Professor, Department of Neurosurgery, NINSH

6. Dr. Md. Farhad Ahmed, Medical Officer, Department of Neurosurgery, NINSH

7. Dr. Sk. Sader Hossain, Professor and Head, Department of Neurosurgery, NINSH

Address of Correspondence: Dr. Kaisar Haroon, Assistant Professor, Department of Neurosurgery, NINS. Email: kaisar298@gmail.com, Phone+01711196577, ORCID: 0000-0002-3065-7877 
Subdural empyemas in adults usually arise from contiguous spread of infection, most often from paranasal sinus infections leading to loculation of pus in the subdural space. Other potential sources of infection leading to SDE are otitis, head trauma, and neurosurgical procedures. ${ }^{4}$

On January 8, 2020, a prescient scientific article was submitted for publication to the Journal of Travel Medicine on a pneumonia of unknown etiology that was identified in patients in Wuhan, China, and the potential for its international spread through commercial air travel. ${ }^{5}$

Coronavirus disease (COVID-19) is an extremely infectious and life-threatening viral illness that gave rise to the current pandemic; an overwhelming healthcare crisis putting the healthcare system under huge strain. COVID-19 outbreak has also brought along disastrous socio-economic effects and heavily impacted healthcare activities including our neurosurgical field. ${ }^{6}$ Neurosurgeons have been sheltered from "frontline action" that is battering subspecialists in emergency, intensive care and pulmonary medicine, but nevertheless, the practice of neurosurgery is yet susceptible to the havoc that COVID-19 has wrought. ${ }^{7}$

The typical clinical manifestation of COVID-19 positive patients are fever, cough, and dyspnea. However, several patients present with neurological symptoms, such as vomiting, headache, dizziness, and delirium. ${ }^{8}$

We are reporting a case of a patient who was suffering from SDE and COVID 19 infection at the same time. We had done literature search through Google Scholar but there was no report of such case.

\section{Case report:}

A 13 years old girl presented to us with the complaints of headache for 14 days, fever for 7 days, convulsion once, blurring of vision for 7 days and vomiting for last 3 days just prior to admission. Her fever was high grade without chill and rigor, it was relieved by taking paracetamol. This happened at the last week of April 2020. The COVID 19 pandemic was highest during that time in Dhaka, Bangladesh. She was suspected of COVID 19 and a sample was sent urgently for RTPCR for COVID 19 which was negative. Her MRI scan of brain with contrast had shown that she was suffering from acute subdural empyema involving the interhemispheric fissure along the parafalcine space. Her GCS was 15 during admission but suddenly her level of consciousness deterioratedand so emergency craniotomy was done. She under went a front parietal craniotomy on the right side. dura was opened and some pus had escaped. The parafalcine space was approached and all pus was evacuated from the interhemispheric space. Thorough toileting with normal saline was done.During surgery it was noted that her brain was very tight and inflamed. So after evacuation of pus the wound was closed without placing the bone flap. The bone was replaces after three months. Then she developed symptoms of fever, malaise, body ache and rhinorrhea and therefore a second sample for RTPCR for COVID 19 was sent. This time report was positive for COVID 19. Learning this, the whole team of care givers was isolated and they underwent test for COVID 19. Of them, 5 neurosurgeons, 3 nurses from Ward and OT, and 2 ward boys were positive.

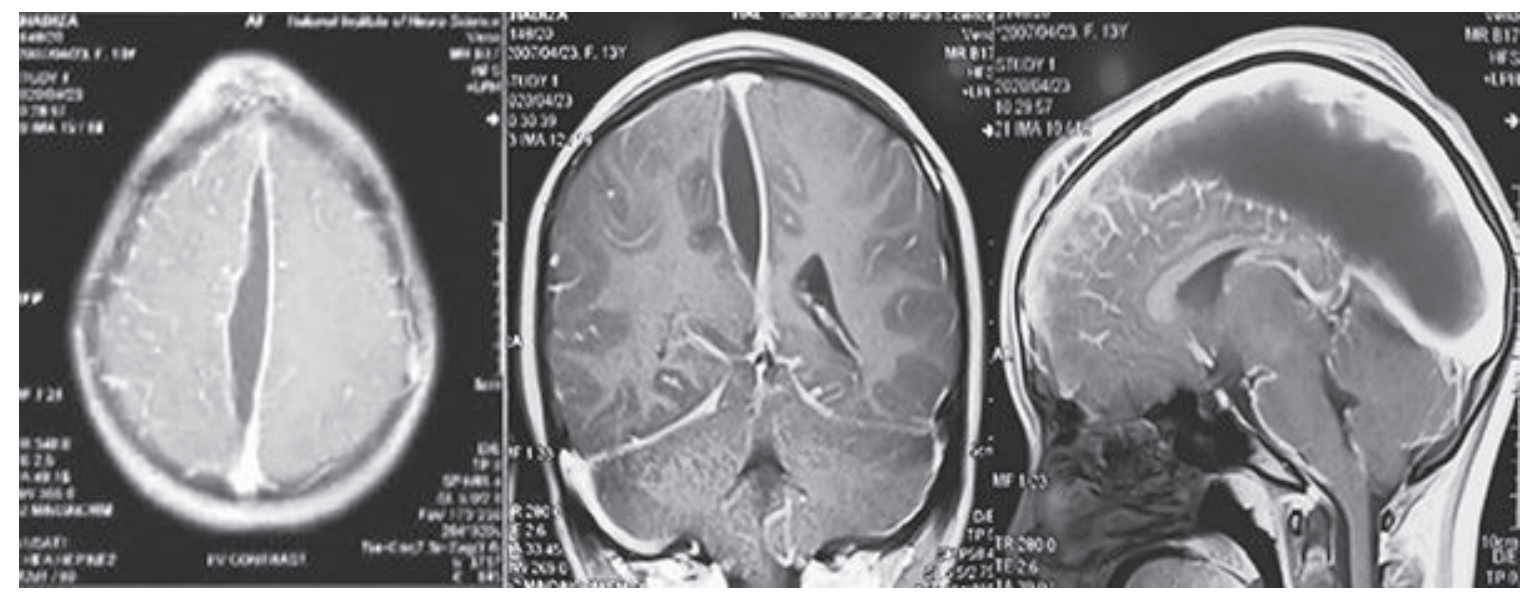

Fig.-1 Contrast MRI of Brain showing abscess in the interhemispheric fissure 


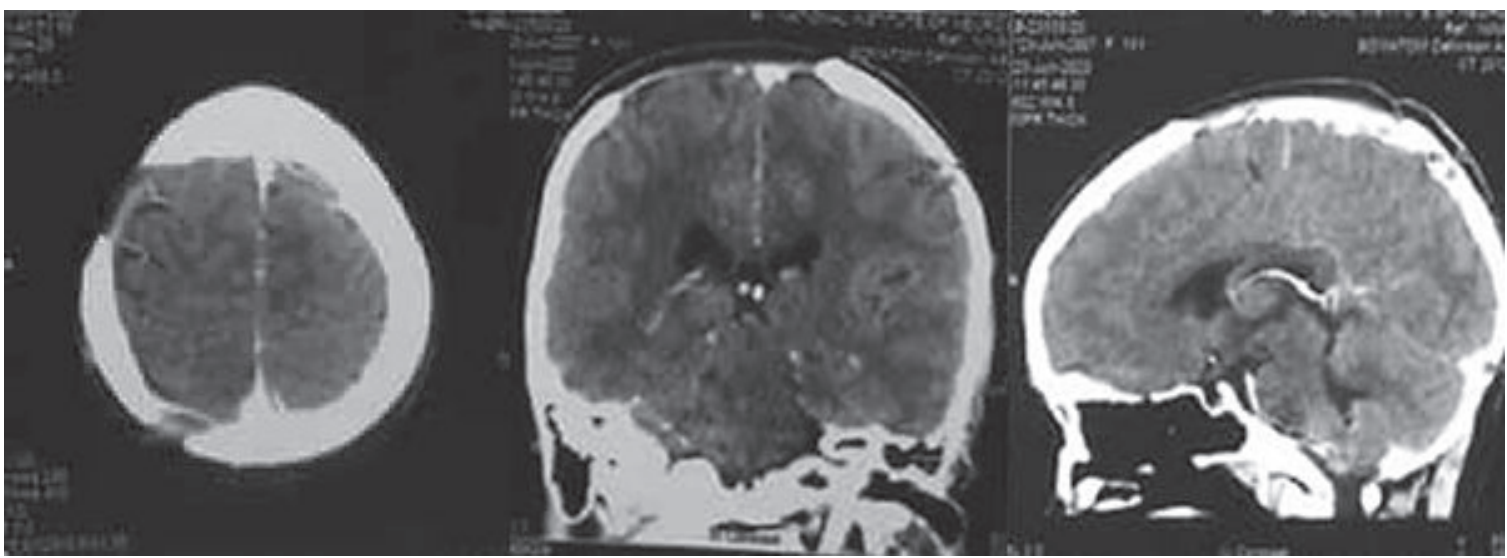

Fig.-2 Post operative CT scan of the same patient after one month

The OT suit had to be fumigated, the general wards had to be disinfected and other patients needed to be separated and tested. The patient herself now had no neurological problem, so she was referred to a dedicated COVID 19 hospital. She had an uneventful recovery and had recovered completely from both COVID 19 and the subdural empyema.

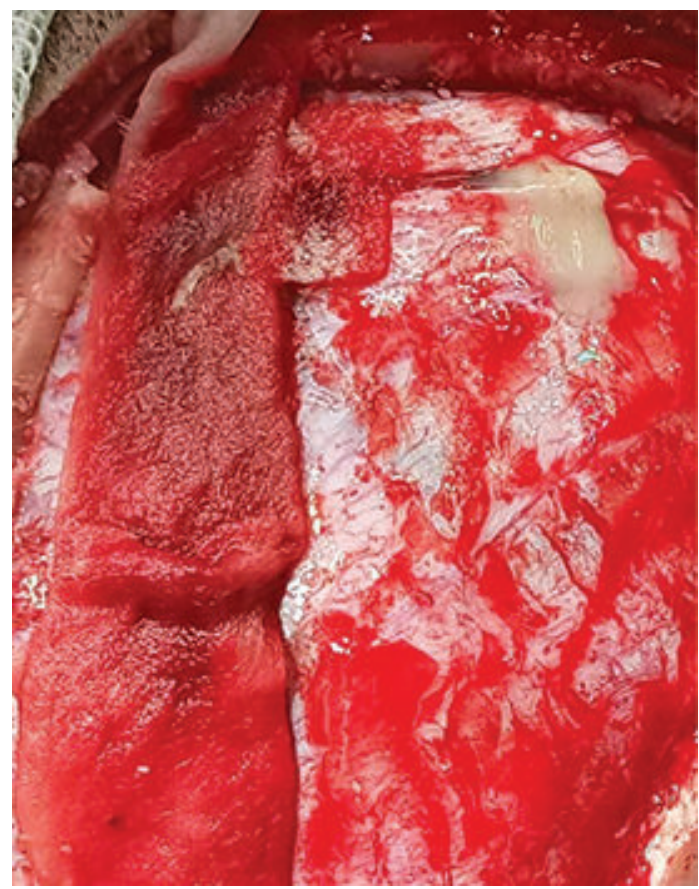

Fig.-3: Pus is coming out after incising the dura

\section{Discussion:}

In our patient the pus was cultured for organisms. There was no growth of organisms in the pus. Though it was sterile, we could not rule out infection due to bacteria or any virus. According to Bernadini, in a large study examining nearly 700 patients with SDE, some of the most common causative organisms, in descending order, include S. milleri group and Streptococcus B. haemolyticus, anaerobic organisms (S. aureus and S. epidermidis, $H$. influenzae, Proteus mirabilus), multiple organisms (two to three) in approximately $15 \%$ of patients, Escherichia coli, and occasionally Pseudomonas aeuroginosa and Klebsiella pneumonia. ${ }^{4}$ But there was noattempt to isolate viruses.

The common belief is SDE is the sequelae of paranasal sinus infection. Again the COVID 19 virus also resides in the PNS. Therefore, it is difficult to say whether the COVID 19 was a sequelae or causation of the parafalcine empyema.

The empyema causes an intense inflammatory reaction in the subdural space; this may be accompanied by CSF pleocytosis and cortical encephalitis Inward venous extension of infection may result in hemorrhagic infarction or superficial abscess. Cerebral edema develops rapidly beneath the empyema, and the combined mass effect of cerebral edema and the empyema leads to transtentorial herniation, brainstem compression, and death. ${ }^{9}$

Early surgical drainage, simultaneous eradication of the primary source of infection, and intravenous administration of high doses of appropriate antimicrobials are the mainstay of treatment for SDE ${ }^{4}$. Craniotomy gave the best survival figures at $91 \%$ for primary, and $81 \%$ for secondary surgery. Burr holes were associated with $52 \%$ survival. ${ }^{3}$ The use of craniotomy coupled with culture and sensitivity based intravenous antibiotics appears to grant the best modality in managing such condition. ${ }^{10}$ In our patient 
we had operated urgently. She recovered completely and without any complications.Factors of prognosis 1) the age of the patient, 2) the source of the infection, 3) the organism cultured, and 4) the surgical procedure employed. ${ }^{3}$

\section{Conclusion:}

Acute subdural empyema is a neurosurgical emergency. It is to beevacuated as soon as it is diagnosed. It is to be seen if there is any association between subdural empyema and COVID 19 in the future. To operate on such a patient is risky for the operating surgeons, attending doctors, nurses and other support staffs.

\section{References:}

1. Prieto R, Ortega C. Parafalcine subdural empyema: The unresolved controversy over the need for surgical treatment. Surgical Neurology International. 2019;10.

2. Bhandari YS, Sarkari NBS. Subdural Empyema. 1970;32(1):35. doi: 10.3171/jns.1970.32.1.0035.

3. Bannister G, Williams B, Smith $\mathrm{S}$. Treatment of subdural empyema. 1981;55(1):82. doi: 10.3171/jns.1981. 55.1 .0082$.
4. Bernardini GL. Diagnosis and management of brain abscess and subdural empyema. Current Neurology and Neuroscience Reports. 2004;4(6):448-56. doi: 10.1007/ s11910-004-0067-8.

5. Kondziolka D, Couldwell WT, Rutka JT. Introduction. On pandemics: the impact of COVID-19 on the practice of neurosurgery. Journal of Neurosurgery. 2020;1(aop):1-2.

6. Lakhdar F, Benzagmout M. Neurosurgery at war with the COVID-19 pandemic: patient's management from an African neurosurgical center. Acta Neurochirurgica. 2020.

7. Jean WC, Ironside NT, Sack KD, Felbaum DR, Syed HR. The impact of COVID-19 on neurosurgeons and the strategy for triaging non-emergent operations: a global neurosurgery study. Acta neurochirurgica. 2020:1-12.

8. Gupta P, Muthukumar N, Rajshekhar V, Tripathi M, Thomas $\mathrm{S}$, et al. Neurosurgery and neurology practices during the novel COVID-19 Pandemic: A consensus statement from India. Neurology India. 2020;68(2):246.

9. Greenlee JE. Subdural Empyema. Current Treatment Options in Neurology. 2003;5(1):13-22. doi: 10.1007/ s11940-003-0019-7.

10. Ahmed MMZE, Alias SA, Ali ME, Mohammed ZI. Surgical Management and Functional Outcome of Primary Convexity Subdural Empyema With Inter-hemispheric Extension: A Case Report and Literature Review. 2020. 